\title{
Towards a geometrical understanding of the CPT theorem
}

\author{
Hilary Greaves
}

November 26, 2007

\begin{abstract}
The CPT theorem of quantum field theory states that any relativistic (Lorentz-invariant) quantum field theory must also be invariant under $\mathrm{CPT}$, the composition of charge conjugation, parity reversal and time reversal. This paper sketches a puzzle that seems to arise when one puts the existence of this sort of theorem alongside a standard way of thinking about symmetries, according to which spacetime symmetries (at any rate) are associated with features of the spacetime structure. The puzzle is, roughly, that the existence of a CPT theorem seems to show that it is not possible for a well-formulated theory that does not make use of a preferred frame or foliation to make use of a temporal orientation. Since a manifold with only a Lorentzian metric can be temporally orientable - capable of admitting a temporal orientation - this seems to be an odd sort of necessary connection between distinct existences. The paper then suggests a solution to the puzzle: it is suggested that the CPT theorem arises because temporal orientation is unlike other pieces of spacetime structure, in that one cannot represent it by a tensor field.

To avoid irrelevant technical details, the discussion is carried out in the setting of classical field theory, using a little-known classical analog of the CPT theorem.
\end{abstract}

\section{Introduction}

A story has it that in the early sixties, Feynman was asked to give an evening talk to physics students at Caltech, explaining the basic idea of the CPT theorem: the celebrated result in quantum field theory that states that any relativistic (i.e. Lorentz-invariant) quantum field theory must be invariant under CPT, the composition of charge conjugation, parity reversal and time reversal. Feynman agreed to commit to doing this, commenting that if one 
cannot explain something to second year Caltech undergraduates then one does not understand it. The story goes that Feynman spent a month or two trying to plan the talk, and then, in despair, cancelled the commitment.

Whether or not this story is true, its basic point is well taken: despite the importance of the CPT theorem in particle physics, the result itself is generally not well understood, even by those whose professional practice regularly appeals to it. It is often referred to as a 'remarkable result'. It seems worthwhile trying to attain a point of view from which the CPT theorem is not remarkable at all, but is, rather, precisely what one expects on elementary grounds. That is the aim of the project of which the present paper is a part.

More precisely, one can identify two positive sources of puzzlement:

- How can it come about that one symmetry (e.g. Lorentz invariance) entails another (e.g. CPT) at all?

- How can there be such an intimate relationship between spatiotemporal symmetries (Lorentz invariance, parity reversal, time reversal) on the one hand, and charge conjugation, not obviously a spatiotemporal notion at all, on the other?

This paper focusses on the first sort of puzzlement. I first sharpen the puzzle by suggesting that, according to a way of thinking about spacetime symmetries that is (for good reason) fairly common currency in the philosophy of physics community, there is a particular reason for thinking that Lorentz covariance should not be able to entail anything like CPT covariance. I then go on to offer a solution to the puzzle.

An outline of the paper is as follows.

Section 2 reviews the standard way of thinking about spacetime symmetries, well discussed by (in particular) Michael Friedman (1983) and John Earman (1989), that will give rise to the sharpened form of our puzzle and that will provide the framework for our discussion. The key point to be taken from this section, for the purposes of this paper, is that one generally expects to find a certain correspondence between the dynamical symmetries of a give spacetime theory, on the one hand, and the spacetime structure postulated by that theory, on the other. More precisely, we expect the following principle to hold: that the covariance group of a theory (when formulated non-generally-covariantly) should, for a well-formulated theory, be equal to the invariance group of the set of geometrical objects that are not represented explicitly in the coordinate-dependent, non-generally-covariant 
formulation in question. (Readers familiar with the standard framework in question can easily skim this section.)

Section 3 suggests that, from this point of view, the existence of a CPT theorem is prima facie puzzling. The idea here will be that a CPT theorem seems to be telling us that it is not possible for a relativistic theory (that is, on our way of thinking, a theory that does not require the existence of a preferred frame or foliation) to make essential use of a temporal orientation. This seems odd, since a temporal orientation - a geometric representation of the direction of time - seems to be a perfectly respectable piece of spacetime structure, and since there is no obstacle to theories' making essential use of other pieces of spacetime structure, such as a metric or a total orientation.

Section 4 states a theorem (proved elsewhere) that seems to be a classical analog of the usual, quantum-field-theoretic, CPT theorem.

Section 5 offers a solution to the puzzle: the key point is that temporal orientation is indeed (in a Lorentzian context) unlike many other pieces of spacetime structure, in that it cannot be represented by a Lorentz-invariant tensor field. Meanwhile, the 'auxiliary constraints' that we expect any 'reasonable' field theory to satisfy have the effect that only pieces of spacetime structure that can be represented by such tensor fields can be made use of in the theory.

Section 6 considers the puzzle and its suggested resolution in the context of Galilean- (rather than Lorentz-) invariant field theories. The point here is that while we do have a 'Lorentzian CPT theorem' - a theorem stating that any Lorentz-invariant field theory must also be CPT invariant - we do not have a 'Galilean CPT theorem' (and there do exist Galilean-invariant, CPT-non-invariant theories). We can therefore perform a 'sanity check' on the discussion of this paper, by checking that the suggested explanation of how anything like a CPT theorem can come about in the Lorentzian case does not also suggest that we should expect to find a CPT theorem in the Galilean case. The result will be reassuring: there is a Galilean-invariant tensor field representing temporal orientation, and, indeed, by making use of the field in question we can easily construct examples of Galilean-invariant, non-CPT-invariant field theories.

Section 7 is the conclusion. 


\section{The connection between dynamical symmetries and spacetime structure}

This section reviews a standard way of thinking about spacetime symmetries. This standard account provides the framework within which the existence of the CPT theorem is, I will suggest, prima facie puzzling. The review in this section is very much in the spirit of the discussions given by Friedman ((1983), chapters 2 and 3) and Earman ((1989), chapters 2 and 3). It may be skimmed by those familiar with the framework in question (the only slightly idiosyncratic elements are the talk of 'special' rather than 'absolute' or 'kinematical' objects, and (relatedly) the terminology 'covariance ${ }_{Q}$ group of a theory'; I indulge in this idiosyncrasy to avoid irrelevant complications concerning how, if at all, one might define 'absolute' of 'kinematical').

Spacetime theories. Let $T$ be a spacetime theory. That is, $T$ is a theory whose intended models are structures of the form $\left\langle M, \Phi_{1}, \ldots, \Phi_{n}\right\rangle$, where $M$ is a differentiable manifold, and the $\Phi_{i}$ are geometrical objects on $M$.

Symmetries. To discuss the symmetries of a theory $T$, we need first to regard the set $M_{D}$ of models of the theory ( $D$ for 'dynamically allowed') as a subset, $M_{D} \subset M_{K}$, of a larger set $M_{K}$ of 'kinematically allowed structures'. We then consider maps from the set $M_{K}$ into itself; we say that such a map is a symmetry of the theory $T$ iff the map leaves the dynamically allowed subset $M_{D}$ invariant.

Spacetime symmetries. We wish formally to capture the sense in which particular groups of spacetime transformations - for example, the Lorentz or Galilei groups - may or may not be symmetries of a given spacetime theory $T$.

There is a familiar obstacle to rendering such talk of spacetime symmetries nontrivial: to finding a sense, that is, in which it is not trivially the case that any manifold diffeomorphism $h: M \rightarrow M$ will count as a 'symmetry of $T$ '. The obstacle arises if, in the structures $\left\langle M, \Phi_{1}, \ldots, \Phi_{n}\right\rangle$, we have explicitly represented all of the structure that is actually presupposed by our theory. It arises as follows. Let $\operatorname{Diff}(M)$ be the set of diffeomorphisms from the spacetime manifold $M$ onto itself. Any such diffeomorphism will have a natural action on a given geometric object $\Phi_{i}$ : for example, if $\Phi_{i}$ is a vector field on $M$, the natural action of $h$ takes $\Phi_{i}$ to its push-forward $h_{*} \Phi_{i}$; if $\Phi_{i}$

is a one-form then the natural action is the pull-back to $h^{*} \Phi_{i}$, etc. Write 
$h * \Phi_{j}$ for the result of allowing $h$ to act in the natural way on $\Phi_{j}$, regardless of the type of geometrical object that $\Phi_{j}$ is (so that we do not have to keep track of distinctions between different types of geometric objects that are irrelevant for our immediate purposes). Now, we wish to associate, with a manifold diffeomorphism $h \in \operatorname{Diff}(M)$, a map $h^{\prime}: M_{K} \rightarrow M_{K}$. The most obvious way to do this is to allow $h$ to have its natural effect on each of the geometrical objects in an arbitrary structure $m \in M_{K}$ : that is, to define $h^{\prime}: M_{K} \rightarrow M_{K}$ by

$$
h^{\prime}\left\langle M, \Phi_{1}, \ldots, \Phi_{n}\right\rangle:=\left\langle M, h * \Phi_{1}, \ldots, h * \Phi_{n}\right\rangle .
$$

However, if we define $h^{\prime}$ in this way, then every $h^{\prime}$ (i.e. the $h^{\prime}$ corresponding to every $h \in \operatorname{Diff}(M)$ ) will be a symmetry of our theory $T$. This is the sense in which, unless there is some structure to $M$ that we have failed to represent in our statement of the models of the theory, any spacetime theory will, trivially, count as generally covariant.

This is unhelpful, since we want to capture the special relationship of, say, the Lorentz group to relativistic electromagnetic theory, and the Galilei group to Newtonian gravitation theory. (One normally wants to say that Newtonian gravitation theory is Galilean-covariant and that Maxwell's equations are not, or that a theory counts as special relativistic just in case it is Lorentz-covariant; we see to be losing an interesting and fruitful distinction if we have only the sense in which all theories are generally covariant.) To do this, we must set up a different correspondence between manifold diffeomorphisms $h$ and maps from $M_{K}$ onto itself, such that in general only for some proper subset of $\operatorname{Diff}(M)$ do we have the corresponding maps as symmetries. Our new correspondence is set up as follows. For a given theory $T$, we single out some subset $Q$ of the $\Phi_{i}$ as 'special'. (One way to go about branding objects 'special' is to look for some formal criterion that will pick some of them out, such as the Anderson-Friedman 'absoluteness' criterion (see, e.g., Friedman ((1983), pp.56-61). Another is to say that the 'special' ones are the 'kinematical' or 'geometrical' ones, and hope that we know what this means. An approach that is less ambitious, but that suffices for our present purposes, is to do without any such general criterion, and simply to specify some subset of the objects in a given theory on a caseby-case basis, putting a subscript on 'covariance' to indicate which set of objects we have chosen to treat as 'special'. Since we don't need to tangle with the problems that the more ambitious programs face, we will take this last approach.) Having chosen our set $Q$, we then write candidate models of $T$ in the form $\left\langle M, S_{1}, \ldots, S_{m}, O_{1}, \ldots, O_{n}\right\rangle$, where the $S_{i}$ ('special' objects) are elements of $Q$ and the $O_{i}$ ('ordinary' objects) are not. We now allow the 
diffeomorphism $h$ to act only on the 'ordinary' objects $O_{i} \notin Q$. That is, to any $h \in \operatorname{Diff}(M)$ we associate a map $h_{Q}: M_{K} \rightarrow M_{K}$, defined as follows:

$$
h_{Q}\left\langle M, S_{1}, \ldots, S_{m}, O_{1}, \ldots, O_{n}\right\rangle:=\left\langle M, S_{1}, \ldots, S_{m}, h * O_{1}, \ldots, h * O_{n}\right\rangle .
$$

We define the covariance $_{Q}$ group of $T$ to be the set of $h \in \operatorname{Diff}(M)$ such that the corresponding $h_{Q}$ is a symmetry of $T$.

The connection between symmetries and spacetime structure. The covariance $_{Q}$ group of a spacetime theory will, in general, be some proper subset of $\operatorname{Diff}(M)$. But more can be said. Define the invariance group of a set $Q$ of geometrical objects as the set of diffeomorphisms $h$ such that (the natural action of) $h$ leaves each element of $Q$ invariant. Suppose it is the case that, for all models of our theory $T$, the invariance group of the set $\left(S_{1}, /\right.$ ldots,$\left.S_{m}\right)$ of 'special' fields appearing in that model is the same. In this case, we can write of the invariance group of $Q$ as a property of the theory, rather than of a particular model of the theory. We then expect that, if our theory $T$ is 'well-formulated', the covariance $Q$ group of $T$ is equal to the invariance group of $Q$.

To support this expectation, we argue first that the covariance $_{Q}$ group of $T$ is a subgroup of the invariance group of $Q$, and then that the invariance group of $Q$ is a subgroup of the covariance $Q$ group of $T$. (Similar arguments are given in Earman (ibid., pp.46-7).)

The first claim - that the covariance $_{Q}$ group of $T$ is a subgroup of the invariance group of $Q$ - follows trivially from the sense in which $T$ is generally covariant. (Since

$$
\begin{array}{r}
\left\langle M, S_{1}, \ldots, S_{m}, O_{1}, \ldots, O_{n}\right\rangle \in M_{T} \\
\Rightarrow\left\langle M, h * S_{1}, \ldots, h * S_{m}, h * O_{1}, \ldots, h * O_{n}\right\rangle \in M_{T},
\end{array}
$$

if in addition we have $h * S_{1}=S_{1}, \ldots, h * S_{m}=S_{m}$, it follows trivially that

$$
\begin{array}{r}
\left\langle M, S_{1}, \ldots, S_{m}, O_{1}, \ldots, O_{n}\right\rangle \in M_{T} \\
\Rightarrow\left\langle M, S_{1}, \ldots, S_{m}, h * O_{1}, \ldots, h * O_{n}\right\rangle \in M_{T} ;
\end{array}
$$

that is, that $h_{Q}$ takes models to models.)

The second claim - that the invariance group of $Q$ is a subgroup of the covariance $_{Q}$ group of $T$ - can arguably be defended, for suitable selections of the set $Q$, by an appeal to Ockham's Razor. Here it is important that the ('special') objects in $Q$ are not themselves 'directly observable' or 'given to 
us by a mechanical experiment': that their existence is, rather, inferred from empirical data that more directly gives us the 'ordinary' objects $O_{i}$. The basic idea is that, if we have a theory whose covariance group is a proper subgroup of the invariance group of $Q$, then it ought to be possible to write down an alternative theory $T^{\prime}$ that has the same empirical consequences as does $T$ as far as the $O_{i}$ are concerned, but that replaces $Q$ with a set $Q^{\prime}$ whose invariance group is smaller than that of $Q$; further, that this alternative theory $T^{\prime}$ is more parsimonious than $T$. The claim then is that, if $T$ is a 'well-formulated' theory (i.e. if $T$ respects Ockham's Razor), the invariance group of $Q$ will be a subgroup of the covariance $Q$ group of $T$.

Non-generally covariant formulations of spacetime theories. While it is often preferable, for the purposes of foundational discussions, to formulate theories in a coordinate-free framework, such a framework is often inconvenient for calculations, and is used in only a minority of the the physics literature. It will therefore be useful to see how the abstract considerations above relate to coordinate-dependent formulations of theories.

When formulating one's theory in a coordinate-dependent way, one faces a choice between two options. (The distinction between the two is precisely analogous to the distinction between the candidate symmetry operations $h^{\prime}$ and $h_{Q}$ given above.) The first option is explicitly to take coordinate components of all the geometrical objects that appear in the coordinateindependent formulation. If one takes this first option, one arrives at a coordinate-dependent formulation that picks out the intended class of models relative to an arbitrary coordinate system. Say that the covariance group of the theory is the group of transformations between coordinate systems that pick out the intended class of models; we thus have, in this first case, $\operatorname{Diff}(M)$ as the covariance group. The second, alternative, option is to represent some chosen subset $Q$ of one's geometrical objects implicitly: that is, to consider its coordinate components as functions of the coordinates, and to 'transform' them, when changing to any other coordinate system, by keeping the same function of the coordinates in the new frame. If one takes this second option, one arrives at a coordinate-dependent formulation that picks out the intended class of models only relative to a certain 'privileged' class of coordinate systems (the 'privileged' class being the class of coordinate systems in which the coordinate components of the implicit geometrical objects happen to be the same as their components in the original, defining, coordinate system); its covariance group will then, in general, be some proper subgroup of $\operatorname{Diff}(M)$, and again we expect that the covariance 
group will be equal to the invariance group of the set $Q$ of objects that we chose to single out for special treatment.

Example. We illustrate the above abstract discussion using the example of special-relativistic electromagnetism. According to this theory, there is a flat Lorentzian metric $g_{a b}$, a tensor field $F_{a b}$ (the electromagnetic field) of type $(0,2)$, and a vector field $J^{a}$ (the charge-current density field). The equations relating these objects are

$$
\begin{aligned}
F_{; b}^{a b} & =-4 \pi J^{a}, \\
F_{[a b ; c]} & =0,
\end{aligned}
$$

where indices are raised using the inverse $g^{a b}$ of the metric, and it is understood that the covariant derivative is the unique one that is compatible with the metric. These equations are generally covariant, in the following two (equivalent) senses:

Coordinate-independent sense of general covariance. If $\left\langle M, g_{a b}, F_{a b}, J^{a}\right\rangle$ satisfies (3) and (4), then so does $\left\langle M, h * g_{a b}, h * F_{a b}, h * J^{a}\right\rangle$, for any manifold diffeomorphism $h: M \rightarrow M$.

Coordinate-dependent sense of general covariance. In coordinate component form, the equations $3-4$ become

$$
\begin{aligned}
F_{\mu \nu ; \nu} \equiv & \frac{\partial F_{\mu \nu}}{\partial x^{\mu}}-\Gamma_{\mu \nu}^{\lambda} F_{\lambda \nu}-\Gamma_{\nu \nu}^{\lambda} F_{\mu \lambda} \\
= & J_{\mu} ; \\
F_{[\mu \nu ; \sigma]} \equiv & \frac{1}{3}\left(\frac{\partial F_{\mu \nu}}{\partial x^{\sigma}}-\Gamma_{\mu \sigma}^{\lambda} F_{\lambda \nu}-\Gamma_{\nu \sigma}^{\lambda} F_{\mu \lambda}\right. \\
& +\frac{\partial F_{\nu \sigma}}{\partial x^{\mu}}-\Gamma_{\nu \mu}^{\lambda} F_{\lambda \sigma}-\Gamma_{\sigma \mu}^{\lambda} F_{\nu \lambda} \\
& \left.+\frac{\partial F_{\sigma \mu}}{\partial x^{\nu}}-\Gamma_{\sigma \nu}^{\lambda} F_{\lambda \mu}-\Gamma_{\mu \nu}^{\lambda} F_{\sigma \lambda}\right) \\
= & 0 .
\end{aligned}
$$

These equations pick out the same (i.e. the intended) class of models in any coordinate system $x: M \rightarrow \mathbb{R}^{4}$.

However, we can also identify a clear sense in which 'the symmetry group of classical electromagnetism' is the Lorentz group, rather than the full diffeomorphism group: 
Coordinate-independent sense of special covariance. Let us single out the metric $g$ as 'special'. Then, for any $h \in \operatorname{Diff}(M)$, we may consider the transformation $h_{g}$, given by

$$
h_{g}\left\langle M, g_{a b}, F_{a b}, J^{a}\right\rangle:=\left\langle M, g_{a b}, h * F_{a b}, h * J^{a}\right\rangle .
$$

For arbitrary $h$, we won't in general expect this transformation to take models to models. In general we'll (instead) expect $h$-covariance $g$ only when $h$ happens to leave $g$ invariant, since, in that case but in that case alone, the RHS of 11 is identical to $\left\langle M, h * g_{a b}, h * F_{a b}, h * J^{a}\right\rangle$. So now we have a nontrivial covariance $g$ group, and it's precisely the group of transformations leaving the 'special' object $g$ invariant: that is, the Lorentz group.

Coordinate-dependent sense of special covariance. If we choose a coordinate system in which the Christoffel symbols vanish (i.e. an inertial coordinate system), then, the equations (5)-(10) reduce, respectively, to

$$
\begin{aligned}
\frac{\partial F_{\mu \nu}}{\partial x^{\nu}} & =J_{\mu} ; \\
\frac{\partial F_{\mu \nu}}{\partial x^{\sigma}}+\frac{\partial F_{\nu \sigma}}{\partial x^{\mu}}+\frac{\partial F_{\sigma \mu}}{\partial x^{\nu}} & =0 .
\end{aligned}
$$

(Noting that

$$
F=\left(\begin{array}{llll}
0 & E_{1} & E_{2} & E_{3} \\
-E_{1} & 0 & -B_{3} & B_{2} \\
-E_{2} & B_{3} & 0 & -B_{x} \\
-E_{3} & -B_{2} & B_{1} & 0
\end{array}\right),
$$

it is straightforward to see that these coincide with usual coordinatedependent form of the Maxwell equations.)

We have gained notational simplicity, relative to (5)-(10), but now we must remember that our equations (12)-(13) pick otu the intended class of models only relative to a privileged class of coordinate systems, which latter are related to one another by Lorentz transformations.

This concludes our review of the standard material within which our puzzle will appear. To sum up the key point of this section: there is an intimate relationship between the spacetime symmetries of a theory, on the one 
hand, and the spacetime structure postulated by that theory, on the other. Specifically, a 'well-formulated' theory fails to have a particular manifold diffeomorphism as one of its symmetries iff it postulates some piece of background ('special') structure that is not invariant under the diffeomorphism in question.

\section{A puzzle about the CPT theorem.}

We are now in a position to state our puzzle concerning the CPT theorem. This theorem states that, subject to some apparently innocuous auxiliary conditions, the following conditional must hold of any quantum field theory $T$ :

If $T$ is invariant under the restricted Lorentz group $L_{+}^{\uparrow}$, then $T$ is actually invariant under CPT.

I mentioned (in the introduction) that it is possible to decompose a general sense of puzzlement at this statement into two parts: one concerning how Lorentz invariance can entail another symmetry at all, and a second concerning how charge conjugation gets into an otherwise spatiotemporal picture. Since our present concern is with the first of these, let us 'pretend' (but justification for this move will be offered in the next section) that, instead of the CPT theorem, we actually a $P T$ theorem. Then we have (instead) the statement

If $T$ is invariant under the restricted Lorentz group $L_{+}^{\uparrow}$, then $T$ is actually invariant under the whole of the proper Lorentz group $L_{+}$(i.e. under the total-reflection component, as well as under the identity component).

In the light of the standard account of spacetime symmetries that I've reviewed, this conditional is prima facie rather puzzling. Here is why. Suppose that we have a theory according to which there are, among other objects, a flat Lorentzian metric $g$, a total orientation $\epsilon$ and a temporal orientation $\tau$. (The total orientation is an object that determines, for any quadruple consisting of one timelike and three (ordered) linearly independent spacelike 4-vectors, whether that quadruple is 'right-handed' or 'left-handed'. It can be represented by a totally antisymmetric rank four tensor, $\epsilon_{a b c d}$. The temporal orientation is an object that specifies in a continuous way, at each point $p$, which is the 'future' lobe of the lightcone in $T_{p} M$. Its possible representations will be considered in section 5.) 
The puzzle is then the following. First, we note the invariance groups of three sets of objects we might choose to treat as 'special' in the sense of section 2:

\begin{tabular}{l|l} 
Special fields & Invariance group $\mathbf{S}_{\mathbf{k}}$ \\
\hline$g$ & $L$ (full Lorentz group) \\
$g, \epsilon$ & $L_{+}$(proper Lorentz group) \\
$g, \epsilon, \tau$ & $L_{+}^{\uparrow}$ (restricted Lorentz group)
\end{tabular}

Here, $L_{+}^{\uparrow}$ is the restricted Lorentz group. This is the set of Lorentz (i.e. $g$-preserving) transformations that can be continuously connected to the identity: it includes all rotations, boosts and products thereof, but does not include parity or time reflection. $L_{+}$is the proper Lorentz group: the set of all metric-preserving Lorentz transformations with determinant one, i.e. the union of $L_{+}^{\uparrow}$ with the set of all Lorentz transformations that reverse both spatial parity and time sense. $L$ is the full Lorentz group: this includes transformations that reverse parity, time sense, both or neither. (See figure 1.)

Ignoring the first of these possibilities (i.e. that of treating $g$ alone as 'special'), we should then expect to be able to write down, not only a non-generally invariant theory whose invariance group is exactly $L_{+}$(by treating $g$ and $\epsilon$ as 'special'), but also a non-generally invariant theory whose invariance group is exactly $L_{+}^{\uparrow}$ (by treating $g, \epsilon$ and $\tau$ as 'special'). A PT theorem, however, tells us that we cannot do the latter: that, subject to the (as yet unstated) auxiliary assumptions of our theorem, we cannot find theories that are invariant under precisely the restricted Lorentz group. It seems to be telling us, that is, that no theory that is 'nice' (in the sense of conforming to these auxiliary assumptions) can actually make use of a temporal orientation, over and above a flat metric and a total orientation. And now one might well wonder why not. Metric, temporal orientation and total orientation seem to be paradigm cases of distinct existences; it's odd to find such necessary connections between them. Or, to put the puzzle another way: where does this discrimination against temporal orientations come from? That is, what feature of temporal orientation can explain why, in the context of the existing objects $g$ and $\epsilon$, they are unusable in this way?

This is not a paradox, but it does seem to be a puzzle whose resolution is likely to be illuminating. In the next section, I give an explicit statement of the theorem that is the source of our puzzle, and in section $5 \mathrm{I}$ offer a resolution. 


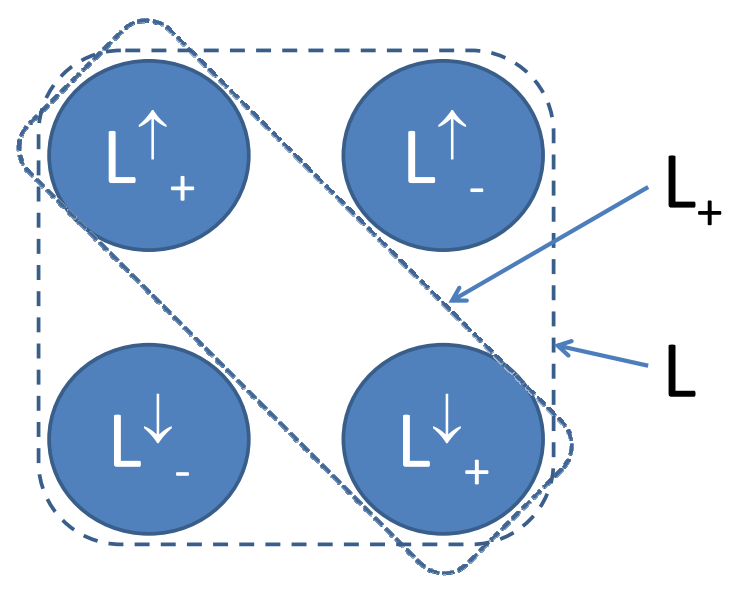

Figure 1: The (real) Lorentz group has four mutually disconnected components, labelled by $\uparrow$ or $\downarrow$ according to whether or not they reverse time sense, and by + or - according to whether or not they reverse total orientation (i.e. whether their determinant is +1 or -1 ). In this notation, $L_{+}^{\uparrow}$ is the 'restricted' Lorentz group; $L_{+} \equiv L_{+}^{\uparrow} \cup L_{+}^{\downarrow}$ is the 'proper' Lorentz group; $L \equiv L_{+}^{\uparrow} \cup L_{-}^{\uparrow} \cup L_{-}^{\downarrow} \cup L_{+}^{\downarrow}$ is the 'full' Lorentz group. 


\section{A classical PT theorem}

At the start of section 3, I promised some motivation for changing the subject from CPT to PT. Here, briefly, are three reasons for going along with that. (The first is pragmatic; the second and third are more justificatory.)

1. The account of the connection between symmetries and 'special fields' has been developed only for spacetime symmetries: transformations that are diffeomorphisms on the spacetime manifold $M$. So PT is a relatively well-understood case to deal with. It's less clear how we're supposed to think about CPT-invariance (i.e. whether, and how, this 'combination of a geometrical and a nongeometrical symmetry' can be associated with the absence of some piece of structure on some larger space). As a research strategy, to avoid unmanageable confusion at the outset, it seems worth starting with the more straightforward case of $P T$, and hoping that the results will generalize to $C P T$.

2. I have growing suspicions that the transformation usually called 'CPT' is actually more properly regarded as $\mathrm{PT}$, that is, as a bona fide spacetime symmetry that is the product of mirror-image reflection and time reversal. If this is right, then I am not actually changing the subject at all, in insisting on talking about PT. But that's another story. ${ }^{1}$

3. Again to avoid irrelevant complications, it will be better to start the foundational discussion in the context of classical (rather than quantum) field theory. And in the classical case, we can prove a theorem that transparently is a $\mathrm{PT}$, rather than a CPT, theorem. (Its precise relationship to the usual quantum 'CPT' theorem is an open question - but it seems inevitable that there will be an intimate connection, and this is part of my reason for thinking that so-called 'CPT' is really just PT.)

The following, then, is a theorem ('classical PT theorem').

\subsection{Bell's theorem}

The following argument is adapted from one given by John S Bell, in a surprisingly little-known paper (Bell, 1955).

In outline, the result is as follows. We consider a classical field theory given by a system of partial differential equations (PDEs) on a specified set

\footnotetext{
${ }^{1}$ This comment relates to work-in-progess by Frank Arntzenius and myself: see, e.g., (Arntzenius \& Greaves, 2007).
} 
of fields. Let $\Phi$ be the space of kinematically allowed fields. (In the general case, we may be dealing with a theory containing a number of interacting fields - scalar fields, tensor fields, etc — so, for a given theory, an element of $\Phi$ will be an ordered $m$-tuple of specified numbers of scalar fields, vector fields, rank 2 tensor fields, etc.) We note that any PDE (with real coefficients, and for real fields) can be expressed as the vanishing of some functional $F: \Phi \rightarrow \mathbb{R}^{M}$ of the fields. (That is, $F$ encodes the dynamics in the sense that: $\phi \in \Phi$ is dynamically allowed iff $F(\phi)$ is the zero map on $M$.) Let there be given a representation of the full Lorentz group $L$ on $\Phi$ (that is, we know how each of our fields 'transforms under Lorentz transformations'). We assume that $F$ is a local polynomial in the fields, and that the fields transform as components of tensors under proper ${ }^{2}$ Lorentz transformations. (Each of these two assumptions is crucial to the theorem.) It can then be proved that, if the set $S$ of solutions of the equation $F(\phi)=0$ is invariant under $L_{+}^{\uparrow}, S$ is actually invariant under the whole of the proper Lorentz group $L_{+}$(i.e. including total reflections as well as rotations and boosts).

Summing this up, the claim is as follows. Let $T$ be a theory according to which there are $n$ dynamical fields $\Phi_{1}, \ldots, \Phi_{n}$. Suppose that the following three conditions hold:

1. The dynamical fields are tensors (of arbitrary rank).

2. The dynamical equations are partial differential equations that are local polynomials in the fields and their derivatives.

3. The set $\mathcal{S}$ of solutions to the dynamical equations is invariant under $L_{+}^{\uparrow}$.

Then, $\mathcal{S}$ is actually invariant under all of $L_{+}\left(\equiv L_{+}^{\uparrow} \cup P T\left(L_{+}^{\uparrow}\right)\right)$.

A formal statement of the theorem behind this claim is given in the Appendix. The proof is given in (Greaves, 2007).

\footnotetext{
${ }^{2}$ That is, the fields may be P-pseudotensors and T-pseudotensors - they may pick up a sign flip, relative to the transformations undergone by true tensors, under parity-reversing and time-reversing transformations — but they may not be PT-pseudotensors, i.e. they must transform as true tensors under total-reflection transformations. The distinction between true tensors and $\mathrm{P} / \mathrm{T}$-pseudotensors is irrelevant for present purposes, since the theorem to be proved deals only with proper Lorentz transformations.
} 


\subsection{Auxiliary constraints}

We were careful, in section 3 , to state our puzzle as arising from the fact that no 'nice' theory is invariant under precisely the restricted Lorentz group, rather than that no theory whatsoever has just that invariance group. 'Nice', here, means 'conforming to the auxiliary assumptions of the PT theorem' (i.e. the conditions (1) and (2) above). It is worth highlighting, then, the fact that these 'innocuous auxiliary constraints' play a crucial role in both the antecedent plausibility, and in the proof, of the PT theorem. There obviously do exist 'theories', in the minimal sense of 'classes of models', that are $L_{+}^{\uparrow}$-invariant but not $L_{+}$-invariant. (To generate one, we need only pick some particular scalar field on $\langle M, g\rangle$ that does not have any interesting symmetries, and take the set that results from closing under the action of the restricted Lorentz group.)

But we would like to know more: we would like to be able to see precisely how it is that the particular auxiliary constraints in question assumptions — which, after all, do look pretty innocuous - manage to rule out the use of a temporal orientation.

In the classical theorem sketched above, we have two auxiliary constraints.

One is a restriction on the types of geometrical objects that the theory postulates: they have to be tensors. This is supposed to rule out, in particular, objects that transform as pseudotensors under PT. If we are allowed PT-pseudotensors, then counterexamples are easy to come by. Here's one: let $\phi$ be a pseudoscalar field, and let the dynamical equation be

$$
\phi=1 \text {. }
$$

Slightly less trivially, suppose that $\psi$ is a scalar field and $\chi$ a pseudoscalar under PT (i.e. $\chi \stackrel{e}{\longmapsto} \chi$ for $e \in L_{+}^{\uparrow}$, but $\chi \stackrel{P T}{\longrightarrow}-\chi$ ). Then, the equation

$$
\psi \chi-\psi=0
$$

is $L_{+}^{\uparrow}$-invariant but not $L_{+}^{\downarrow}$-invariant.

Our second auxiliary constraint is a restriction on the dynamics: the dynamics must express the vanishing of all members of some particular set of polynomials in the coordinate components of the fields and their derivatives. There are two points here that are worthy of note. The first is that some equations, fairly plausible from the point of view of physics, are not 'polynomial' in the required sense. ${ }^{3}$ It may be possible to weaken the assumptions of the theorem, so as to cover these cases also. The second point

\footnotetext{
${ }^{3}$ For example, equations involving terms like $\sin \left(\nabla_{a} \phi \nabla^{a} \phi\right)$ or operators like $\sqrt{\nabla^{2}+m^{2}}$
} 
is that some 'dynamics' do not express the vanishing of any mathematically simple functional $F$ at all (polynomial or otherwise). One example of this phenomenon is given by the 'theory' sketched at the start of this subsection; another is given by the theory 'Inc' stated below (see footnote 5).

Be this as it may, there still seems to be something prima facie puzzling even about the restricted claim that all theories within the stated class obey the conditional 'if $L_{+}^{\uparrow}$-invariant then $L_{+}$-invariant' - there is no connection yet apparent between the restrictions involved in the assumptions of the theorem on the one hand, and the surprising ineffectiveness of temporal orientation on the other. This is the puzzle we wish to solve.

\section{Resolution of the puzzle}

Let us take stock. We started (section 2) by sketching a way of thinking about spacetime symmetries according to which the set of dynamical symmetries ought to coincide with the invariance group of a set of objects that we have (for some reason or none) decided to single out as 'special'. We then noted (section 3) that, on this way of thinking, a PT theorem seems to be asserting that, subject to apparently innocuous auxiliary constraints, it is not possible to write down a theory that makes essential use of a temporal orientation, over and above a Lorentzian metric and a total orientation, and that this is puzzling. To ground the discussion, we then gave (in section 4) a statement of such a theorem, for the case of classical field theory. We now seek a more enlightened point of view: a point of view from which the existence of such theorems in certain cases is not puzzling at all, but is, rather, precisely to be expected, where and only where they in fact occur.

My suggestion is that the following observation lies at the heart of the otherwise puzzling nature of the CPT theorem: there is no tensor field that represents temporal orientation and no more, in the context of a flat Lorentzian metric and a total orientation.

The remainder of this section has two aims. The first is to explicate this observation - what exactly it means, and why it is true. The second is to explain how this helps to dissolve the puzzle. It will be easiest to tackle both of these aims simultaneously.

Intuitively, a temporal orientation on a (temporally orientable ${ }^{4}$ ) manifold $M$ is supposed to specify which temporal direction is 'the future'. Let $p$ be an

are not 'polynomial'. I am grateful to Robert Geroch and Michael Kiessling (resp.) for pointing out these particular examples.

${ }^{4}$ Definition: A manifold $M$ equipped with a Lorentzian metric $g$ is said to be temporally orientable iff there exists a continuous, nowhere-vanishing, timelike vector field on $M$. 
arbitrary point in a temporally orientable manifold $M$ that is equipped with a Lorentzian metric $g$. Then, the tangent space $T_{p} M$ can be divided into timelike, spacelike and null vectors. Further, the set of timelike vectors in $T_{p} M$ has two disconnected components: these will be the 'past' and 'future' lobes of the lightcone at $p$ ('will be' rather than 'are', because until and unless we have a temporal orientation, neither lobe is distinguished as the 'future' one).

Now, we wish to represent temporal orientation by some geometric object on $M$. Here we have a choice: there are many structures on $M$ that would do the trick.

The most obvious way (perhaps) of representing temporal orientation is by a map that assigns, to each point $p \in M$, one of the two lightcone lobes in $T_{p} M$ (and that does so in a continuous way, i.e. the assignments of lightcone lobes to neighboring points must be 'mutually consistent'). This is our first candidate way of representing temporal orientation.

But let us now recall the use we wish to make of our pieces of spacetime structure: we wish to formulate laws that relate other ('dynamical'/'matter') fields to them, so that, by treating the spacetime structures as 'special', we can restrict the invariance groups of non-generally-invariant formulations of our theories. We then note that, if, as seems to be usually the case, our physical laws take the form of differential equations coupling various geometrical objects to one another, then a 'map from spacetime points to lightcone lobes' is not an object we can easily work with. The point is that if $f$ is such a map, the idea of a 'differential equation for $f$ ' does not seem to make sense; $f$, that is, is not the right sort of object to appear in differential equations.

This observation suggests a second possible way of representing temporal orientation. Instead of using a map from spacetime points to lightcone lobes, we could use a continuous nonvanishing timelike vector field, $t^{a}$, on $M$. (We can then pick out the 'future'-directed timelike vectors $v^{a} \in T_{p} M$ as those that have positive 'dot product' $g_{a b} v^{a} t^{a}$ with $t^{a}$ (relative to a convention according to which the metric has signature $(+,-,-,-)$ rather than $(-,+,+,+))$.) This move solves the problem we faced when trying to make use of $f: t^{a}$, as a vector field, is an object of a type that we perfectly well know how to use in differential equations. However, we have now incurred

Heuristically: iff a manifold $M$ fails to be temporally orientable, then one can 'paralleltransport' a timelike vector $v$ at some point $p \in M$ around the manifold, and return to the point $p$ with a vector $v^{\prime}$ that points in the opposite temporal direction to $v$. In this case, it is not possible to make any continuous global specification of which temporal direction is 'the future'. 
a problem of a different sort: $t^{a}$ is not restricted-Lorentz invariant. That is, it is not the case that, $\forall l \in L_{+}^{\uparrow}, l * t^{a}=t^{a}$. The point here is that $t^{a}$ picks out more structure than we wanted to pick out: we wanted only to pick out a preferred lobe of the lightcone at each point, but a vector field picks out, in addition, a preferred timelike vector in the chosen lightcone lobe. The upshot of this is that when we combine our 'temporal orientation' $t^{a}$ with or existing pieces of structure $g_{a b}, \epsilon_{a b c d}$, we do not have a set whose invariance group includes $L_{+}^{\uparrow}$ : rather, the most we expect is the group of translations and rotations (if $g_{a b}$ is flat and $v^{a}$ is constant).

This observation suggests a third possible way of representing temporal orientation: rather than a single (continuous nowhere-vanishing timelike) vector field, we could take an equivalence class of such vector fields (where $s^{a} \sim t^{a}$ iff $g_{a b} s^{a} t^{b}>0$ ). But now we are back to our original problem: an equivalence class of vector fields, as opposed to a particular vector field, is not the right sort of object to appear in a single partial differential equation. ${ }^{5}$

More generally: suppose we convince ourselves that the geometric objects we can make use of, in equations that satisfy the restrictions we have laid down, are just those that can be represented by tensor fields. ${ }^{6}$ Then,

\footnotetext{
${ }^{5}$ Such an equivalence class of vector fields can, of course, be used to generate a set of differential equations. Here is a non-PT-invariant theory that makes use of this idea: Take the temporal orientation $\tau$ to be the set of all nowhere vanishing, future-directed timelike vector fields. Let there be (besides the temporal orientation, total orientation and metric) a single scalar field $\phi$. Say that $\phi$ is dynamically allowed iff the following condition holds:

(Inc) There exists at least one vector field $v^{a} \in \tau$ such that, at every spacetime point $p \in \mathbb{R}^{4}, v^{a} \nabla_{a} \phi>0$.

(This theory is cooked up to say, in a restricted-Lorentz-invariant way, ' $\phi$ increases towards the future', and hence not to be $P T$-invariant.)

This example shows that the restrictions on the dynamics that appear in the premises of the theorem include restrictions on the 'logical form' of the dynamics: it's crucial to the theorem that the sort of existential quantification that's going on in this example is disallowed.

${ }^{6}$ It is not entirely clear that this is true. For example, the covariant derivative is usually thought of as a map from tensor fields of type $(n, m)$ to tensor fields of type $(n, m+1)$, and not itself as a tensor field; and yet it can be used in PDEs. This suggests that perhaps the present discussion must be extended to some class of geometric objects that is wider than the class of tensor fields. However, it is also true that the covariant derivative can be represented by a tensor field (viz. the metric - since the covariant derivative operator is uniquely determined by the metric), so perhaps not.

A second point in this vein is that I am ignoring the issue of density weight. When one writes "tensor" rather than "tensor density of weight $n$ ", one normally implies that the object under discussion has density weight zero. I do not intend this implication. Density weight is irrelevant for present purposes, since all the transformations under consideration have determinant unity. (For an explanation of the concept of density weight, see, e.g.,
} 
we can avail ourselves of the following mathematical fact:

There does not exist any Lorentz-invariant tensor field with an odd number of spacetime indices. ${ }^{7}$

It is easy to see, meanwhile, that a tensor representing the temporal orientation would have to have an odd number of spacetime indices; to represent temporal orientation, it must not be invariant under PT, but any tensor with an even number of spacetime indices is invariant under PT.

We can then conclude, as stated, that no Lorentz-invariant tensor field can represent temporal orientation; given our conviction, it follows that no Lorentz-invariant theory can make use of a temporal orientation.

\section{Galilean-invariant field theories}

We now wish to perform a sanity check on the suggestion of section 5, by considering the case of Galilean-invariant field theories.

The point here is that the PT theorem does not hold in the Galilean case. That is, the following hypothesis is false:

Galilean PT hypothesis. If $T$ is a spacetime theory containing tensor fields, whose dynamics are polynomial in the fields and their derivatives, and if in addition $T$ is invariant under the restricted Galilean group $G_{+}^{\uparrow}$, then $T$ is PT-invariant.

Therefore, if our suggested explanation of the possibility of a Lorentzian PT theorem is on the mark, it had better not be the case that the analogous

$(?, ?)$, pp. 23-5.)

${ }^{7}$ Proof: We first note the existence of a type $(0,2)$ and of a type $(2,0)$ restrictedLorentz-invariant tensor, namely, the Minkowski metric $\eta_{a b}$ and its inverse $\eta^{a b}$. Second, we note that there does not exist a nonzero restricted-Lorentz-invariant tensor of type $(0,1)$ or of type $(1,0)$. (This latter is easy to prove by considering a coordinate system — which always exists - in which such a tensor has the form $(a, 0,0,0)$ — and noting that, since there is always a restricted Lorentz transformation that nontrivially mixes the first coordinate with the second, it cannot be that such an object has the same coordinate representation in every nonreflected inertial coordinate system.)

Now, consider an arbitrary tensor $T^{a_{1} \ldots a_{n}} b_{1} \ldots b_{m}$, with $n+m$ odd; we will show that $T$ cannot be restricted-Lorentz-invariant. To show this, suppose WLOG that $n$ is even, $m$ odd. Define a new tensor $T^{\prime}$, of type $(0,1)$, as follows:

$$
\left(T^{\prime}\right)_{b}:=\eta_{a_{1} a_{2}} \ldots \eta_{a_{n-1} a_{n}} \eta^{b_{1} b_{2}} \ldots \eta^{b_{m-2} b_{m-1}} T_{b_{1} \ldots b_{m-1} b}^{a_{1} \ldots a_{n}}
$$

Then, if $T$ were restricted-Lorentz-invariant, $T^{\prime}$ would be too. But this is impossible, since we have already shown that there are no rank 1 restricted-Lorentz-invariant tensors. 
statement is also true in the Galilean case. That is, it had better not also be true that there is no way of representing temporal orientation against a background of Galilean spacetime structure, without 'picking out more structure than we want', i.e., in this case, the object that represents temporal orientation had better be invariant under the restricted Galilean group.

At first sight, things look worrying. One of the points we met in the Lorentzian case was that a vector field picked out a timelike direction, as well as a privileged direction of time. But privileged timelike directions are no more acceptable in the Galilean setting than in the Lorentzian.

Fortunately for our suggested explanation, however, it does not, in fact, also go through in the Galilean case. There is no Galilean-covariant vector field, but there is a Galilean-covariant one-form (corresponding to the fact that, in Galilean spacetime, there is no preferred timelike direction, but there is a priveleged notion of simultaneity). In this section we explain this point, and we use it to develop a counterexample to the Galilean PT hypothesis.

\subsection{Temporal orientation in Galilean spacetime}

One encodes the structure of Minkowski spacetime using a flat Lorentzian metric $g$; elements of the (full) Lorentz group are then transformations leaving $g$ invariant. Things are less simple in the Galilean case: there is no single geometric object, as it were, that will encode, in a single shot, all of the structure of Galilean spacetime.

Let us first get clear about what the structure is that we are trying to encode, over and above topological and differential structure. To model the Galilean case, we want our spacetime to possess a natural foliation into a family of three-dimensional hypersurfaces, the preferred simultaneity slices. We want each simultaneity slice to be equipped with a Euclidean spatial metric. We want there to be a fact, for any two points of spacetime, about what is the (absolute value of the) temporal distance between them. And we want there to be a fact, for any timelike curve, about whether or not it is 'straight' (i.e. is an inertial trajectory). Iff we want to endow our Galilean spacetime with a temporal orientation, then we also want there to be a privileged total ordering on the set of simultaneity slices.

One way of encoding the aspects of this structure, aside from temporal metric and temporal orientation, is as follows (here I largely follow Friedman ((1983), pp. 71-92), who sets this approach out in far more detail). We start, as in the Lorentzian case, with a four-dimensional differentiable manifold $M$. The affine structure (i.e. the set of facts about which lines in the spacetime are 'straight') is encoded by a connection $\Gamma$. The Euclidean spatial metrics 
are encoded by a rank 2 tensor field $h^{a b}$.

We now face the question of how to encode the temporal metric and/or temporal orientation. Suppose first that we wish to encode temporal metric without picking out a preferred temporal orientation. This can be done by means of a symmetric tensor field of type $(0,2)$ (satisfying certain restrictions; cf. Earman (1989), pp.30-31; in Earman's notation, the tensor field in question is $h_{i j}$ ). This object will tell us the temporal distance between any two time-slices, but will not tell us which is to the future of which. Second, though, suppose that we do wish to encode a temporal orientation, in addition to a temporal metric. Then, we can use a one-form, $t_{a}$ (this can be thought of as the exterior derivative, $t_{a}:=(d t)_{a}$, of a global time function $t$ that respects the simultaneity structure in the sense that the surfaces of constant $t$ are the simultaneity surfaces). This represents temporal metric and temporal orientation at once, in the natural way: if $v^{a}$ is a timelike vector, then $\left|t_{a} v^{a}\right|$ is the temporal length of that vector, and the sign of $t_{a} v^{a}$ tells us whether $v^{a}$ is future- or past-directed. And $t_{a}$ can be chosen to be invariant under the restricted Galilean group $G_{+}^{\uparrow}$, so we have not picked out more structure than we wished to encode.

\subsection{Counterexample to the Galilean PT hypothesis}

The above suggestion for encoding temporal orientation in Galilean spacetime, via the one-form $t_{a}$, can easily be used to generate a counterexample to the 'Galilean PT hypothesis' above. Here is one such counterexample:

Suppose we have a theory containing a scalar field $\phi$ and vector field $\chi^{a}$, whose dynamics are given in generally covariant form by single equation

$$
t_{a} v^{a}=h^{a b} \phi_{; a ; b}
$$

Here, $t_{a}, h^{a b}$ are understood as, respectively, the temporal structure and Euclidean spatial metric structure outlined above for Galilean spacetime.

Suppose now that we treat $t_{a}$ and $h^{a b}$, and in addition the a flat connection $\Gamma$, as 'special'. Then, we have a privileged class of coordinate systems: the inertial frames in which $t$ increases towards the future. In these coordinate systems, the dynamics is given by the non-generally-covariant equation

$$
v^{0}=\nabla^{2} \phi
$$


Under a restricted Galilean transformation, both $v^{0}$ and $\nabla^{2} \phi$ are invariant. However, under $\mathrm{PT}, \nabla^{2} \phi$ is invariant while $v^{0}$ flips sign. Hence, PT in general does not take solutions to solutions, while restricted Galilean transformations do. So this theory constitutes a counterexample to the Galilean PT hypothesis.

\section{Conclusions}

The existence of a PT theorem (such as that discussed in this paper) is prima facie puzzling, since it seems to show that a reasonable theory cannot make use of a temporal orientation, over and above a flat Lorentzian metric and total orientation, without also using extra, 'unwanted' structure such as a preferred frame. One might well wonder where this discrimination against temporal orientations comes from. This paper has suggested that temporal orientation in a relativistic context indeed is special, as pieces of spacetime structure go: it cannot be represented by a tensor field. Meanwhile, we seem to be committed to constraining principles on our physical theories (e.g. constraints on the types of PDEs theories may use), such that structure that cannot be encoded via tensor fields (or 'similar') cannot be used. This dissolves the puzzle.

Several open questions remain. The most pressing is perhaps the following. The discussion above was carried out in the classical context, using a 'classical PT theorem'. It is an open question whether the same sort of line of thought can be used to illuminate the CPT theorem in quantum field theory. This would, presumably, require first reconstruing the operation of charge conjugation as a geometrical operation on a par with parity and time reversal, so that the 'CPT' theorem is also, properly understood, a PT theorem. I am hopeful that this project can be completed. One then wonders whether similar lines of thought can also illuminate the relationship between parity violation and ' $\mathrm{CP}$ ' violation, the possibility of $\mathrm{CPT}$ violation, and so on.

\section{A Classical PT theorem}

A more precise statement of the theorem referred to in section 4 follows. The proof is given in (Greaves, 2007).

Theorem 1 (Classical PT theorem for polynomial systems of real tensor fields.). Let $M$ be a differentiable manifold that is topologically $\mathbb{R}^{4}$. 
Let $\Phi$ be a space of $n$-tuples of tensor fields of specified types on $M$. That $i s$, suppose there is a fixed set of integers $n_{i}, m_{i}: i=1, \ldots, n$ such that each $\phi \in \Phi$ is an $n$-tuple of the form $\left(\phi_{1}, \ldots, \phi_{n}\right)$, where, for each $i, \phi_{i}$ is a tensor field of type $\left(n_{i}, m_{i}\right)$.

Let $\eta$ be a flat Lorentzian metric on $M$. Let $L$ be the group of manifold diffeomorphisms $l: M \rightarrow M$ leaving $\eta$ invariant (i.e., $L$ is the Lorentz group). Let $L_{+}^{\uparrow}, L_{+}^{\downarrow}, L_{-}^{\uparrow}, L_{+}^{\downarrow}$ be the connected subsets of $L$ that reverse neither time sense nor parity, time sense but not parity, parity but not time sense, and both time sense and parity respectively. For arbitrary $l \in L$ and $\phi \in \Phi$, let $l \phi$ be the $n$-tuple of fields obtained by allowing $l$ to act in the natural way on each element of $\phi$.

Let $F: \Phi \rightarrow \mathbb{R}^{M}$ be a functional that is polynomial in the fields and their derivatives. That is, suppose there exists a chart $x: M \rightarrow \mathbb{R}^{4}$, non-negative integers $p, q$ and real coefficients $\left\{a_{m_{1}, \ldots, m_{n}} \in \mathbb{R}: m_{1}, \ldots, m_{n}=0, \ldots, p\right\}$ such that for all $\phi \in \Phi$,

$$
F(\phi)=\sum_{m_{1}, \ldots, m_{n}=0}^{p} a_{m_{1}, \ldots, m_{q}}\left(\psi_{1}\right)^{m_{1}}\left(\psi_{2}\right)^{m_{2}} \ldots\left(\psi_{q}\right)^{m_{q}},
$$

where each $\psi_{j}$ is a specified partial coordinate derivative (possibly zeroth order) of a specified one of the $\left(\phi_{i}\right)^{\mu_{1} \ldots \mu_{n_{i}}} \nu_{1} \ldots \nu_{m_{i}}$, and multiplication is defined pointwise in the obvious way. ${ }^{8}$

Let $S \subseteq \Phi$ be given by

$$
S:=\{\phi \in \Phi: F(\phi)=0\}
$$

[the intended interpretation being that $S$ is the set of solutions to the partial differential equation expressed by the condition $F=0$ ].

Suppose that $S$ is invariant under $L_{+}^{\uparrow}$, i.e. for any $\phi \in S$ and $l \in L_{+}^{\uparrow}$, $l \phi \in S$ also. Then, $S$ is actually invariant under all of $L_{+}$.

\section{References}

Arntzenius, F., \& Greaves, H. (2007). Time reversal in classical electromagnetism. (Draft available from http://philsci-archive.pitt.edu)

Bell, J. S. (1955). Time reversal in field theory. Proceedings of the Royal Society of London A, 231 (1187), 479-95.

\footnotetext{
${ }^{8}$ We note that if this condition holds relative to one global coordinate chart on $M$, it holds relative to all of them. Hence, the 'polynomial' character of $F$ does not pick out any privileged proper subset of coordinate systems.
} 
Earman, J. (1989). World enough and space-time. Cambridge, MA: MIT Press.

Friedman, M. (1983). Foundations of space-time theories. Princeton: Princeton University Press.

Greaves, H. (2007). Classical 'CPT theorem'. (Forthcoming.) 